\title{
Démarreur-testeur par ordinateur via le port USB d'un PC pour moteurs asynchrones triphasés
}

\author{
J. MBIHI \\ Email : mbihidr@yahoo.fr \\ E. AMIE EBANDA et A. DONWOUNG KANA \\ Groupe de Recherche en Informatique Industrielle et Automation (GR2IA) \\ École Normale Supérieure d'Enseignement Technique (ENSET), \\ B.P. 1872, Douala, Cameroun.
}

\begin{abstract}
Résumé
Le démarreur-testeur USB pour moteurs asynchrones triphasés présenté dans cet article, est un système didactique, initié et développé au sein du Groupe de Recherche en Informatique Industrielle et Automation (GR2IA). Il permet aux étudiants aux de niveau Master en génie électrique et informatique de l'ENSET de Douala, d'expérimenter les concepts de base de la commande des processus par ordinateur. Son originalité est fondée en partie sur trois aspects innovateurs : Interface USB, trois modes de démarrage exécutables à partir d'un panneau de contrôle virtuel, et rétroaction des états logiques réels de l'appareillage de puissance (relais thermique, contacteurs), ce qui facilite les opérations de maintenance à distance. L'ensemble du démarreur-testeur et de son logiciel d'application développé en Visual Basic, constitue un banc d'essais d'appoint, qui offre un rendement pédagogique énorme comparé aux démarreurs classiques de moteurs asynchrones triphasés.
\end{abstract}

Mots clés : Démarreur-testeur USB, moteurs asynchrones triphasés, commande par ordinateur, panneau de contrôle virtuel, appareillage de puissance, banc d'essais.

Niveau des étudiants : Le banc d'essais à vocation didactique présenté dans cet article, est utilisé par les étudiants de niveau Master de l'ENSET de Douala, dans le cadre d'une première étude de cas complète, en commande par ordinateur des processus. Il peut aussi être utilisable avec succès au $1^{\mathrm{e}}$ cycle EEA si l'étude de la partie logicielle est limitée aux fonctionnalités du code exécutable.

Niveau de connaissances requis : Schémas électriques, programmation système.

\section{Introduction}

Trois principaux types de dispositifs d'entraînement pour moteurs asynchrones triphasés sont utilisables dans l'industrie. On distingue :

- Les démarreurs électriques à commande manuelle ou à commande semi-automatique par boutons poussoirs [1].

- Les variateurs électroniques à microprocesseurs ou par ordinateur ([2] et [Int 1]).

- Les démarreurs programmables [3].

Le premier type de démarreurs cité est dédié à la plupart de systèmes d'entraînement en boucle ouverte (ventilateurs, agitateurs, tapis roulants, manutention, concasseur, broyeurs, etc.), alors que le second type convient aux systèmes d'entraînement régulés en position ou en vitesse (traction, usinage, fabrication robotisée, etc.). Les démarreurs de troisième type dits 
programmables, sont des démarreurs électriques à commande numérique par PIC (Programmable Integrated Circuit ou circuit intégré programmable) ou par ordinateur. C'est le cas du démarreur initié dans [3] à commande par ordinateur via le port LPT d'un PC.

Le nouveau prototype de démarreur-testeur USB décrit dans cet article, a été développé au sein du Groupe de Recherche en Informatique Industrielle et Automation (GR2IA) de l'ENSET de Douala. En le connectant à un PC contenant le programme d'application prévu, on obtient une plate-forme d'appoint permettant aux étudiants de Master technologique en génie électrique et génie informatique de l'ENSET de Douala, de découvrir et d'expérimenter les nouvelles technologies de commande par ordinateur des processus. Le démarreur proposé combine la technologie électrique et la logique programmable par ordinateur via l'interface USB d'un PC, et se distingue des démarreurs existants par les possibilités innovatrices suivantes :

- Flexibilité : Il offre plusieurs modes de démarrage automatiques (Etoile, Triangle et Etoile-Triangle), avec un seul mode de marche actif à la fois.

- Sécurité : Le changement d'un mode de démarrage en cours est entièrement sécurisé de façon logicielle, sans re-câblage ni de l'interface de puissance, ni de l'interface de commande électrique.

- Instrumentation virtuelle: Le logiciel de commande offre un panneau virtuel de dialogue opérateur.

- Surveillance à distance: L'état logique de l'appareillage de la partie puissance s'affiche en temps réel au panneau de dialogue opérateur, ce qui simplifie les opérations de télémaintenance.

- Temporisation en mode Etoile-triangle modifiable à volonté (au niveau du panneau de dialogue opérateur), et réalisable de façon logicielle à l'aide d'un Timer système.

Les prochaines sections de l'article permettront de comprendre l'architecture, le fonctionnement et l'utilisation didactique du nouveau type de démarreur proposé. Ces sections sont organisées comme suit :

- Partie matérielle du démarreur-testeur.

- Logiciel du démarreur-testeur.

- Mode opératoire et résultats d'essais.

- Conclusion.

- Références bibliographiques.

- Figures citées dans le texte.

\section{Partie matérielle du démarreur-testeur USB}

Le schéma synoptique de la partie matérielle du démarreur-testeur USB proposé est représenté à la figure 1.

Figure 1. Schéma synoptique du banc d'essais du démarreur-testeur USB

La figure 2 illustre le schéma de principe du prototype de banc d'essais du démarreur-testeur USB, où le module VM110 désigne une carte USB isolée, sans alimentation électrique de puissance. Elle a été acheté chez Velleman/Gotronic.

Figure 2. Schéma de principe du banc d'essais du démarreur-testeur USB 
Une prise de vue de cette carte correspond à la figure 3. Par ailleurs, un module d'alimentation externe à sortie continue de 24 Volt s'est avéré nécessaire pour la commande des relais à partir des sorties digitales de la carte USB-VM110.

\section{Figure 3. Prise de vue de la carte USB-VM110 utilisée}

Du point de vue opérationnel, le schéma de principe du banc d'essais (figure 2) montre que :

- Les trois sorties digitales Do1, Do2 et Do3 de l'interface USB sont utilisées pour la commande respective des relais KAD, KAY et KAL. Ces relais commande respectivement à leur tour les contacteurs respectifs KMD, KMY et KML.

- Les quatre entrées digitales Di1, Di2, Di3 de l'interface USB sont utilisées pour la lecture des états logiques des contacteurs respectifs KMY, KML et KMD.

- L'entrée digitale Di4 permet de lire l'état du contact à fermeture RT/ 97-98 du relais thermique RT en vue de détecter tout éventuel défaut. Un tel défaut aurait en même temps pour conséquence, une coupure d'alimentation des circuits de commande des contacteurs, et ce à cause de l'ouverture du contact RT/ 97-98 du relais thermique RT. Pendant toute la durée du défaut, les systèmes d'alimentation de la carte USB et des relais KAD, KAL et KAY sont tout à fait opérationnels, ce qui rend aisé l'automatisation du processus d'initialisation complet de la partie commande.

- Les états logiques réels des entrées-sorties digitales de l'interface USB, sont déportées au niveau du panneau de dialogue opérateur, via un câble USB.

Pour des raisons didactiques, le banc d'essais complet du démarreur-testeur est constitué de sous-ensembles séparés comme le montre la figure 4.

\section{Figure 4. Composantes du banc d'essais du démarreur-testeur USB}

On distingue :

- Un ordinateur PC (figure 4(a)) ayant un port USB et contenant le logiciel d'application développé spécifiquement pour notre produit.

- Interface USB (figure 4(b)): relié d'un côté au port USB d'un PC et de l'autre à l'interface de commande digitale. Le lecteur intéressé peut consulter les normes de conception et de construction des produits USB dans [Int. 2]. Cette interface est constituée de :

○ Une carte USB VM112-K8055 de Velleman/Gotronic ([Int-3], [Int-4], Int-5]) qui a été choisie en raison de ses fonctionnalités modestes et de son prix raisonnable. Elle a été livrée avec ses documents techniques respectifs ([4], [5]) de montage et de programmation, et avec son pilote utilisable pour le développement d'applications avec Visual Basic, Builder $\mathrm{C}++$, Delphi, etc. Chacune des 8 sorties digitales de cette carte est de type collecteur ouvert et supporte jusqu'à $50 \mathrm{~V}-100 \mathrm{~mA}$. Elle admet aussi 5 entrées digitales auxquelles on peut connecter des capteurs logiques. Cette interface séparée peut être utilisée pour contrôler d'autres types de processus.

- Un bloc d'alimentation de la carte USB VM112- K8055, réalisé sur place et monté avec cette carte dans un coffret fabriqué à cet effet.

- Interface de commande digitale (figure 4(c)), reliée d'un côté à l'interface USB et de l'autre à l'interface de puissance. Elle a été réalisée à l'aide de relais électromagnétiques dont le courant nominal des bobines d'excitation peut être supporté par le circuit de sortie à collecteur ouvert de la carte USB VM112- K8055. 
- Interface de puissance (figure 4(d)), reliée d'un côté à l'interface de commande électrique et de l'autre au système d'alimentation électrique triphasé, et admettant les bornes assorties pour le raccordement des enroulements du stator d'un moteur asynchrone triphasé. Elle a été réalisée à l'aide de l'appareillage électromécanique classique (01 sectionneur porte-fusible et 03 contacteurs) de puissance maximal 3 KW sous $220 / 380 \mathrm{~V}$ et $50 \mathrm{~Hz}$;

- Un moteur asynchrone triphasé de $220 / 380 \mathrm{~V}, 50 \mathrm{~Hz}$ à 6 bornes de couplage statorique $U, V, W$ et $X, Y, Z$. (figure 4(e)).

Les informations détaillées au sujet de la réalisation et de la mise en service de la maquette didactique de l'interface USB complète, sont détaillées dans [6]. Les maquettes didactiques des autres modules d'interface restants ont été construites et bien testées ultérieurement.

\section{Logiciel du démarreur-testeur USB}

\subsection{Fonctions du logiciel}

Le logiciel du démarreur-testeur USB offre les fonctionnalités suivantes :

- Choix du mode de marche désiré (Etoile, Triangle, Etoile-Triangle, Arrêt).

- Saisir la valeur de la temporisation désirée en mode Etoile-Triangle.

- Exécution du mode de marche choisi.

- Scanner à chaque $100 \mathrm{~ms}$ et afficher l'état de l'appareillage de puissance (contacteurs, relais thermique).

- Arrêt du mode de marche en cours manuellement, ou automatiquement en cas de déclenchement du relais thermique.

- Sortie de l'application.

\subsection{Panneau de dialogue opérateur}

Après avoir esquissé sur papier un panneau de contrôle conforme aux spécifications définies dans la section 3.1, nous l'avons implémenté avec Visual Basic en suivant les techniques de programmation système développées dans [3]. Étant donné que la carte d'interface USB VM112-K8055 a été livrée avec une bibliothèque dynamique K8055D.DLL contenant les fonctions de communications et d'accès à ses entrées-sorties matérielles, il a donc été nécessaire de déclarer dans le programme source Visual Basic de la feuille de l'application, les lignes de code commentées suivantes :

\footnotetext{
Private Declare Function OpenDevice Lib "k8055d.dll" (ByVal CardAddress As Long) As Long 'Ouvre le pilote

Private Declare Sub CloseDevice Lib "k8055d.dll" ( ) Ferme le pilote

Private Declare Sub ClearDigitalChannel Lib "k8055d.dll" (ByVal Channel As Long)_ 'Mise à 0 d'1 sortie digitale

Private Declare Sub ClearAllDigital Lib "k8055d.dll" () 'Mise à 1 des sorties digitales

Private Declare Sub SetDigitalChannel Lib "k8055d.dll"_ 'Mise à 1 d'l sortie digitale (ByVal Channel As Long) '6

Private Declare Function ReadDigitalChannel Lib "k8055d.dll" (ByVal Channel As Long) As Boōean 'Lecture d'un canal

Private Declare Function ReadAllDigital Lib_ "k8055d.dll" () As Long 


\section{Mode opératoire et résultats d'essais}

Le mode opératoire d'une séance de travaux pratiques portant sur la commande de moteurs asynchrones par démarreur-testeur USB est le suivant :

\subsection{Préparation des travaux pratiques}

1) Étude des entrées-sorties de la carte USB-VM112.

2) Etude de l'architecture complète et du fonctionnement des autres paries matérielles du banc d'essais.

3) Étude des fonctions utiles de la bibliothèque de liens dynamique K8055D.DLL fournie par le fabriquant de la carte USB-VM112.

4) Connaissance des codes Visual Basic des modes de démarrage.

\subsection{Installation et initialisation des interfaces de commande}

1) Branchement (par câble USB) de l'interface USB d'un côté à l'ordinateur contenant le logiciel de démarrage proposé, et de l'autre à l'interface de commande digitale.

2) Branchement de l'interface de commande digitale (précédemment relié à l'interface USB) à l'interface de puissance à vide (sans le moteur).

3) Lancement du logiciel d'application installé dans l'ordinateur, puis ouverture de la communication avec la carte USB en cliquant sur le bouton de commande connexion, et initialisation des sorties digitales de la carte USB à l'aide du bouton de commande Arrêt.

4) Mise sous tension de l'interface USB, et de l'interface de puissance puis fermeture du sectionneur.

\subsection{Essais sans le moteur}

1) Sélection d'un mode de marche au panneau de dialogue opérateur : Etoile, Triangle ou Etoile-Triangle.

2) Clic sur le bouton Marche pour lancer le mode sélectionné, d'où l'observation sur le panneau de contrôle de l'état de l'appareillage puissance.

3) Clic sur le bouton Arrêt pour l'arrêter le mode de marche en cours.

4) Test de tous les modes de marche .

\subsection{Essais de démarrage d'un moteur asynchrone triphasé}

1) Branchement du moteur à l'interface de puissance.

2) Test de tous les modes de marche avec le moteur.

3) Clic sur le bouton Quit pour sortir du logiciel de démarrage.

Les figures 5 à 9 illustrent les exemples de copies d'écran des résultats d'essais obtenus à divers stades du déroulement des travaux pratiques de commande d'un moteur asynchrone par démarreur-testeur USB.

Figure 5. Couplage automatique en Etoile.

Figure 6. Couplage automatique en triangle. 
Figure 7. Couplage automatique en Etoile-Triangle.

Figure 8. Défaut dî à l'ouverture du relais thermique.

Figure 9. Changement des modes de marche sécurisé.

\section{Conclusion}

Le démarreur-testeur présenté dans ce travail permet aux étudiants de niveau Master de génie électrique et génie informatique de l'ENSET de Douala d'expérimenter aisément et rapidement trois modes de démarrage assistés par ordinateur d'un moteur asynchrone triphasé. Pendant l'expérimentation, le passage d'un mode de marche au suivant est sécurisé, flexible et se fait sans re-câblage du système de commande. Il offre donc un rendement pédagogique énorme dans un environnement de travail à la fois convivial, hautement flexible, et fort apprécié par les utilisateurs cibles. Compte tenu des possibilités didactiques optimales offertes par le premier prototype de démarreur-testeur USB en service à l'ENSET, nous envisageons de chercher un financement, qui permettra d'étendre l'utilisation de cette nouvelle génération de produit, à une vaste communauté universitaire, voire industrielle.

\section{Références bibliographiques}

[1] P. BOYE et A. BIANCIOTTO, "Le Schéma en électrotechnique", Delagrave, 1981.

[2] DELTALAB HYDRO TECHNIC, "Automatismes: Platine - variateurs multifonctions pour moteur asynchrone avec ou sans codeur, MV1004", Catalogue de bancs didactiques de DELTALAB.

[3] J. MBIHI, "Informatique et Automation: Automatismes programmables contrôlés par ordinateur". Editions Publibook Universités, Paris, 2006.

[4] Velleman/Gotronic, "K8055_VM110 USB board - Kit assembly and information manual_K8055_FR". Manuel de montage de composants.

[5] Velleman/Gotronic, "K8055_VM110 USB boardlSoftware ManuallMan_FR_K8055 DLL". Manuel de programmation.

[6] E. AMIE EBANDA et A. DONWOUNG KANA, "Commande par ordinateur des processus séquentiels via l'interface USB". Mémoire de fin d'études en vue de l'obtention du DIPET-II, proposé et dirigé par J. MBIHI, GR2IA, ENSET de Douala, 2006.

\section{Sites Internet intéressants}

[Int-1] www.deltalab-cosimi.fr

[Int-2] http://www.usb.free.com.

[Int-3] www.gotronic.fr

[Int-4] www.velleman.com

[Int- 5] www.vellemanusa.com 
Figures cités dans le texte

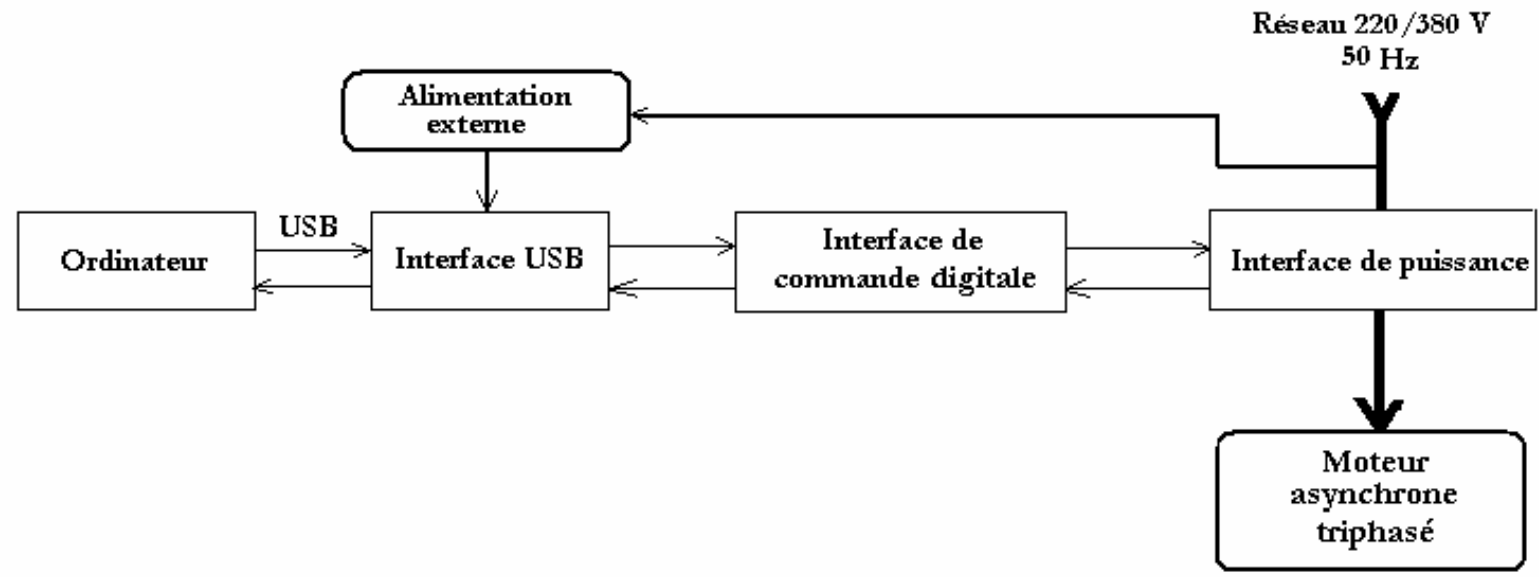

Figure 1 Schéma synoptique du banc d'essais du démarreur-testeur USB

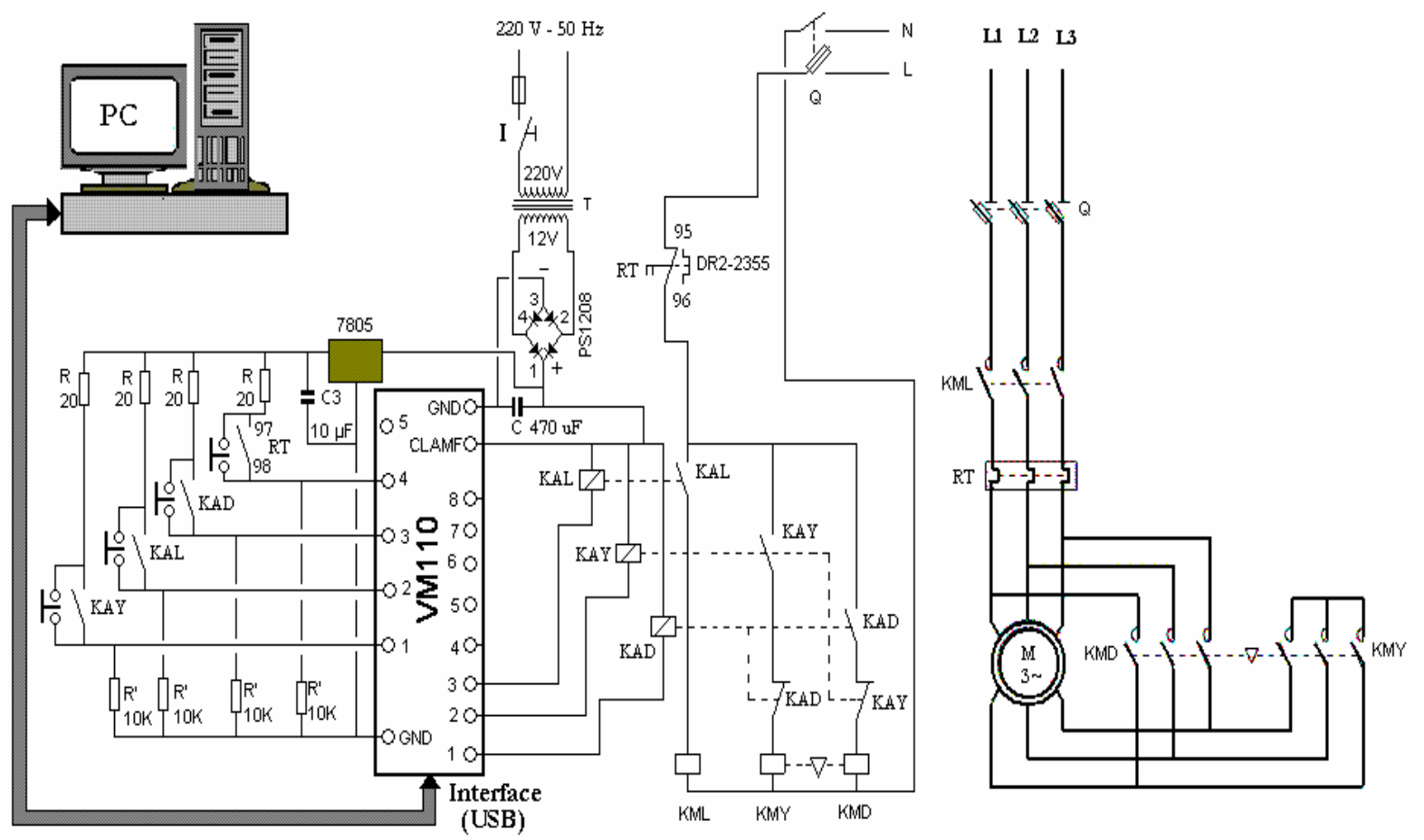

Figure 2 Schéma de principe du banc d'essais du démarreur-testeur USB 


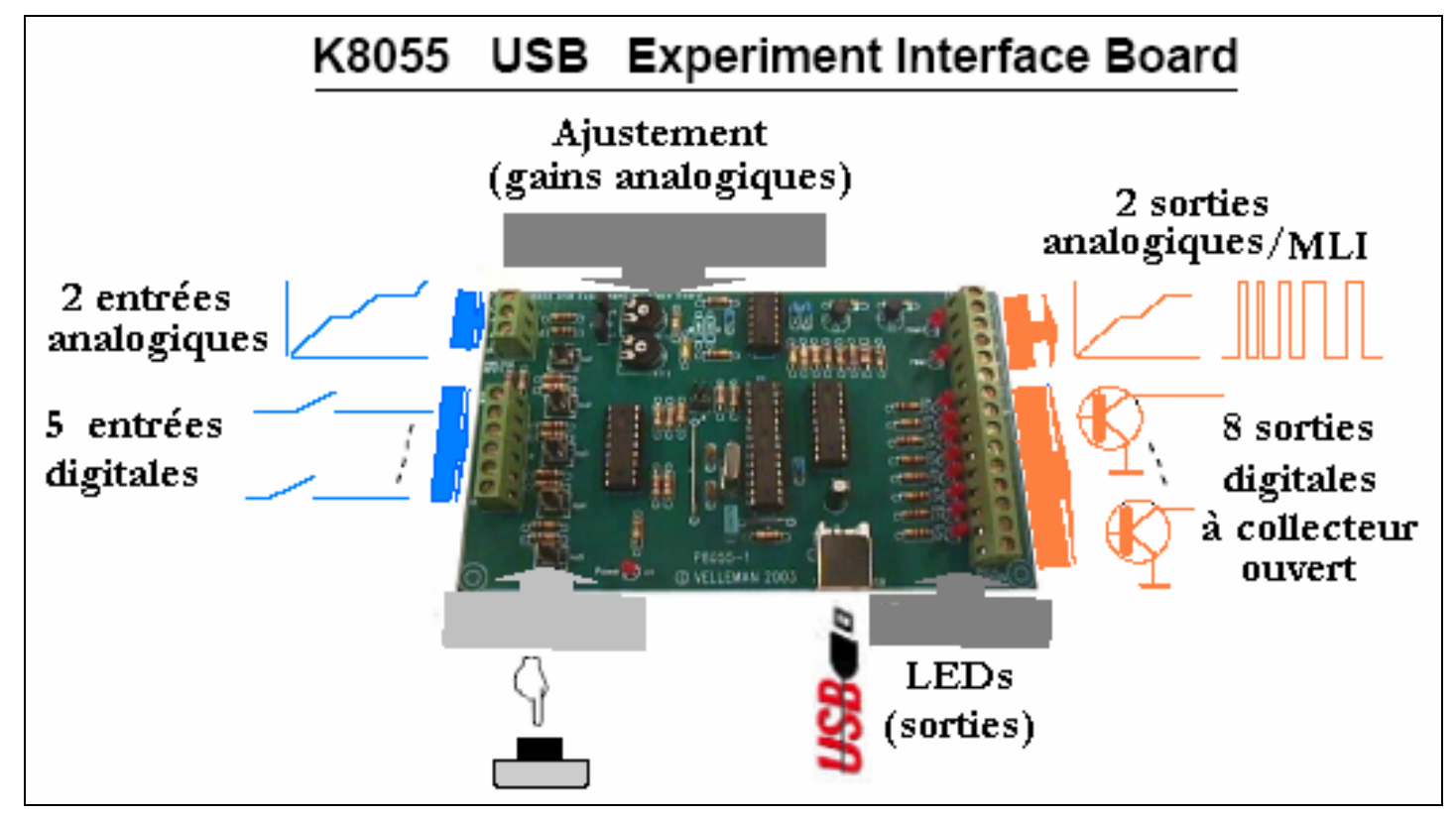

Figure 3 Prise de vue de la carte USB utilisée

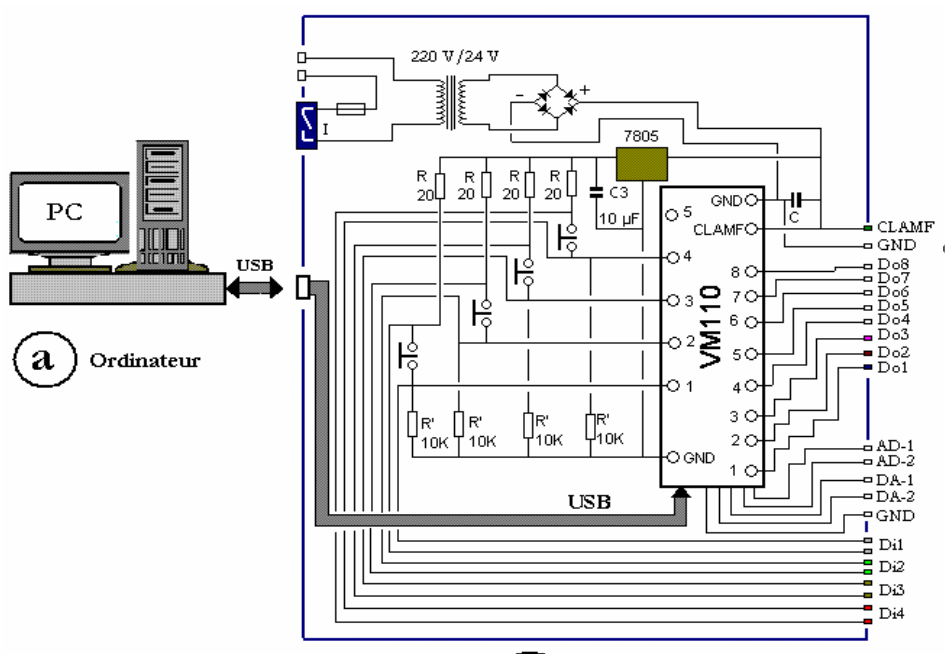

(b) Interface USB
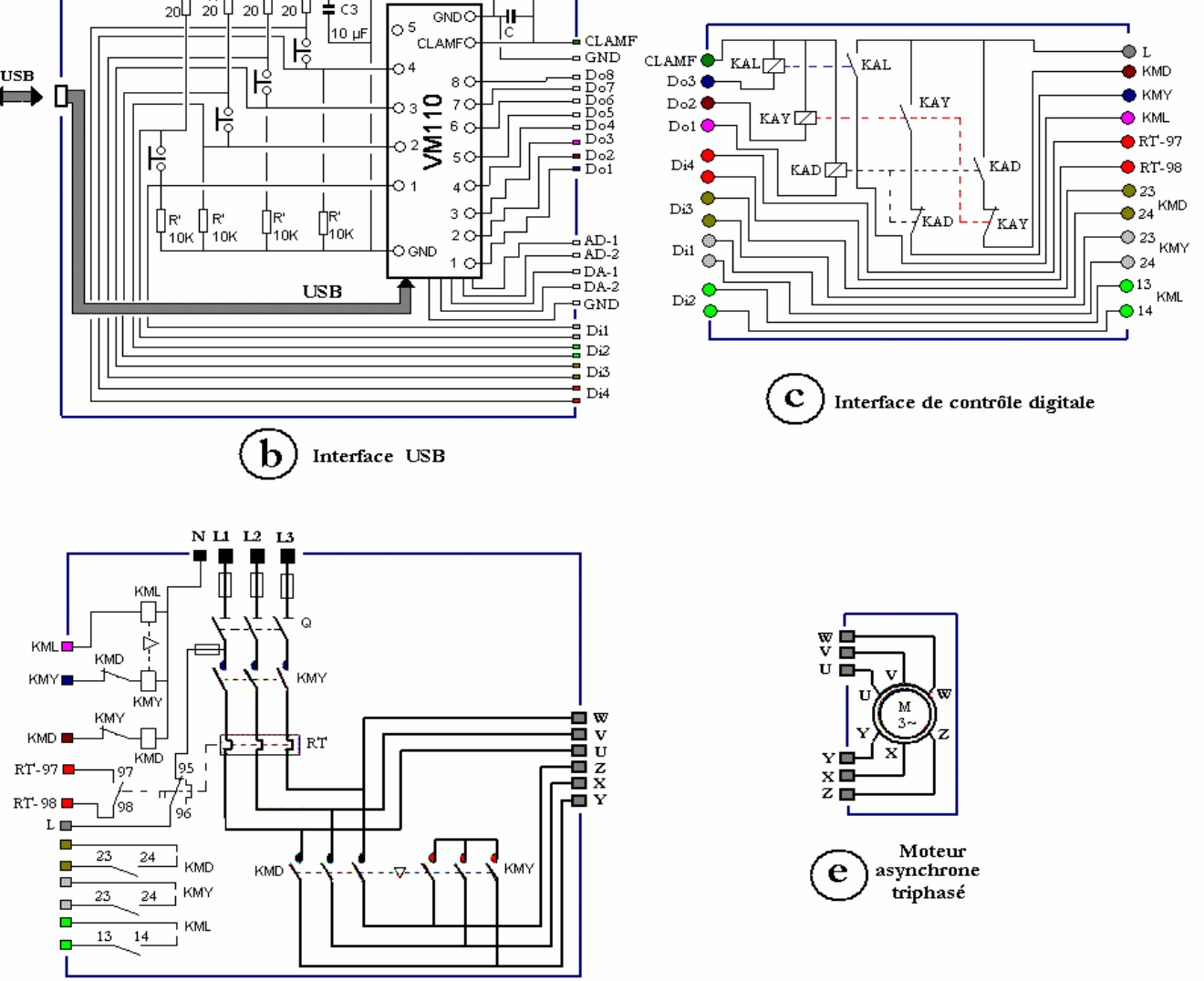

(d) Interface de puissance

Figure 4 Composantes du banc d'essais complet du démarreur-testeur USB 


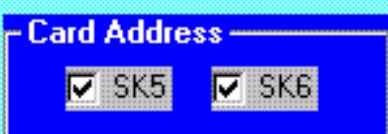

Connect

Card 0 connected

MODES DE MARCHE

O Etoile

\section{Triangle}

Etoile-Triangle

Tempo [s] 3
SORTIES VERS - Appareillage de puissance

KAL KAY KAD

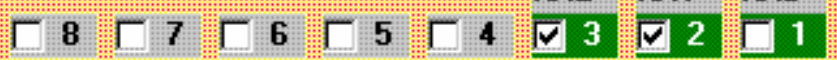

ENTREES - Etat réel Appareillage
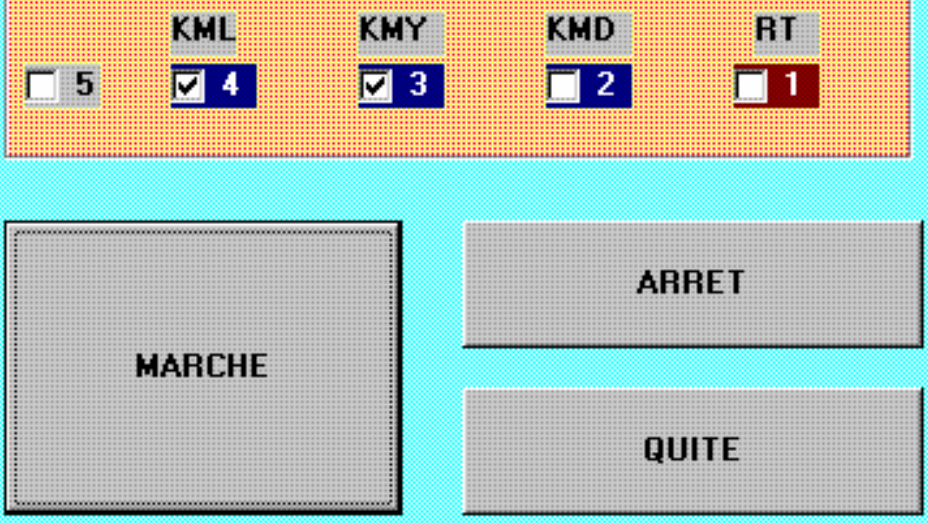

Figure 5 Démarreur-testeur USB : Couplage automatique en Etoile

Démarreur USB Flexible/GR2IA

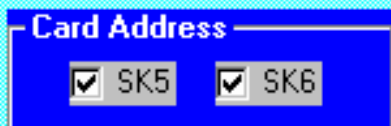

Connect

Card 0 connected

MODES DE MARCHE

Etoile

\section{Triangle}

Etoile-Triangle

T empo [s] 3
SORTIES VERS - Appareillage de puissance

KAL KAY KAD

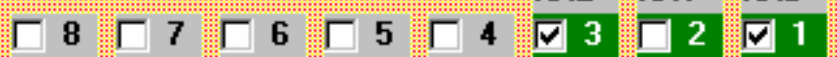

ENTREES - Etat réel Appareillage

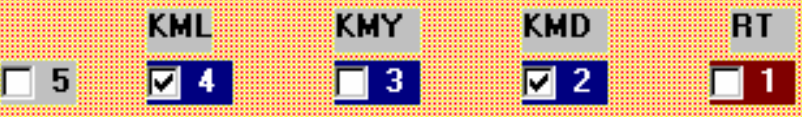

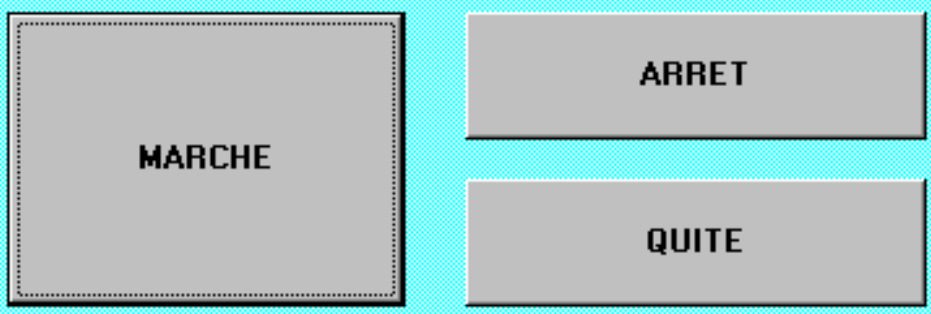

Figure 6 Démarreur-testeur USB : Couplage automatique en triangle 


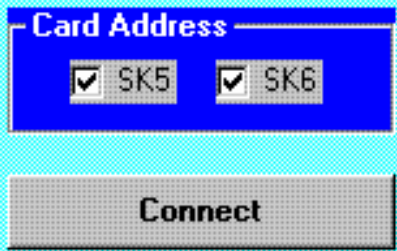

Card 0 connected MODES DE MARCHE

Etoile

Triangle

\section{Etoile-Triangle}

T empo [s] 3
SORTIES VERS - Appareillage de puissance

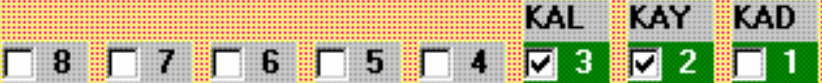

ENTREES - Etat réel Appareillage

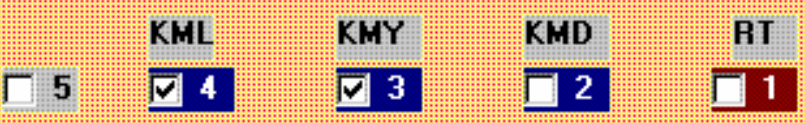

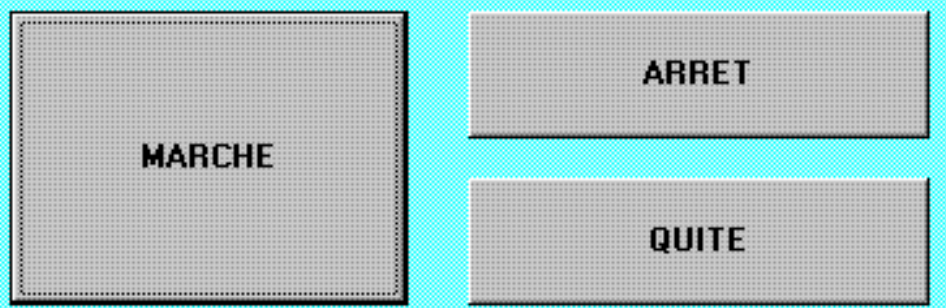

(a) Phase Etoile

a, Démarreur USB Flexible/GR2IA

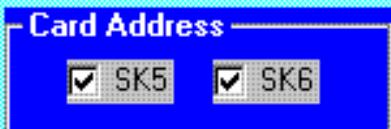

Connect

Card 0 connected

MODES DE MARCHE

Etoile

\section{Triangle}

Etoile-Triangle

T empo [s] 3

SORTIES VERS - Appareillage de puissance

$$
\text { Г } 8 \text { ए }
$$

ENTREES - Etat réel Appareillage

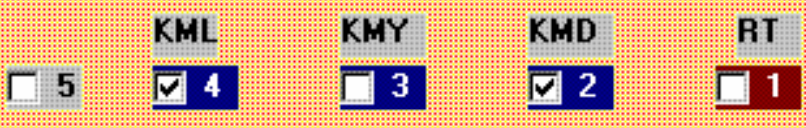

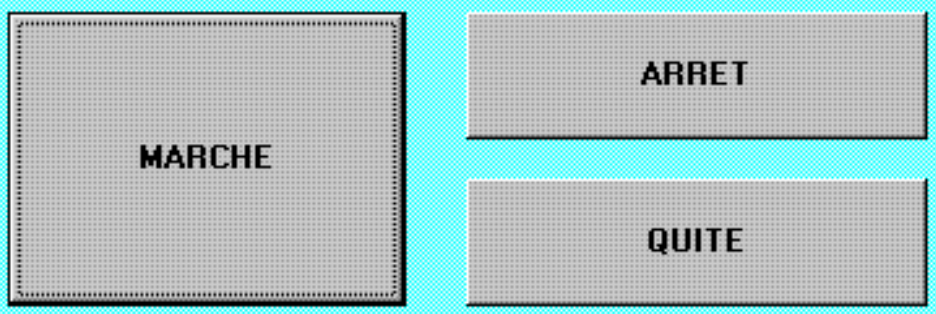

(b) Phase triangle

Figure 7 Démarreur-testeur USB : Couplage automatique en Etoile-Triangle 


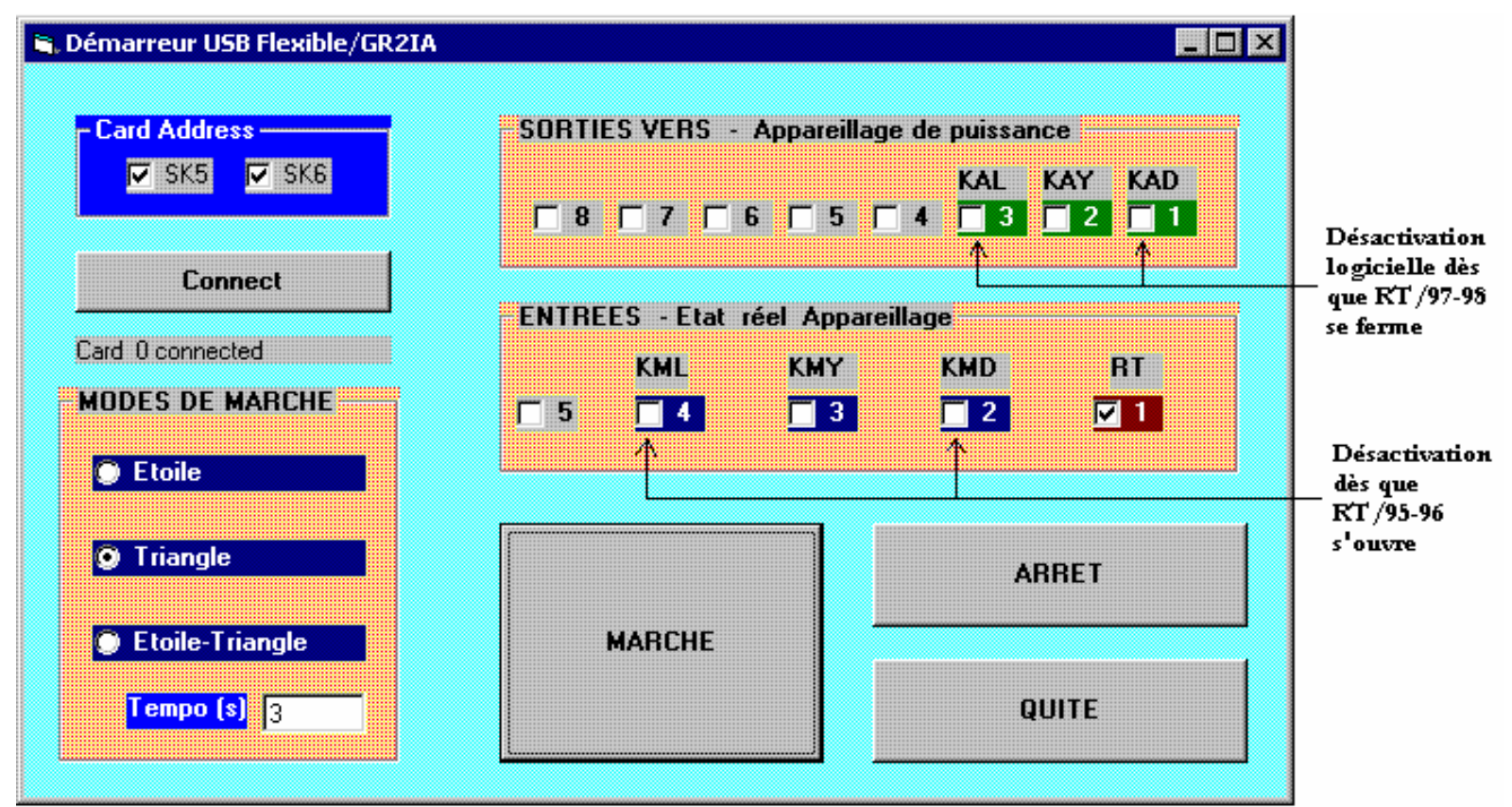

Figure 8 Démarreur-testeur USB lors d'un défaut dî à l'ouverture du relais thermique

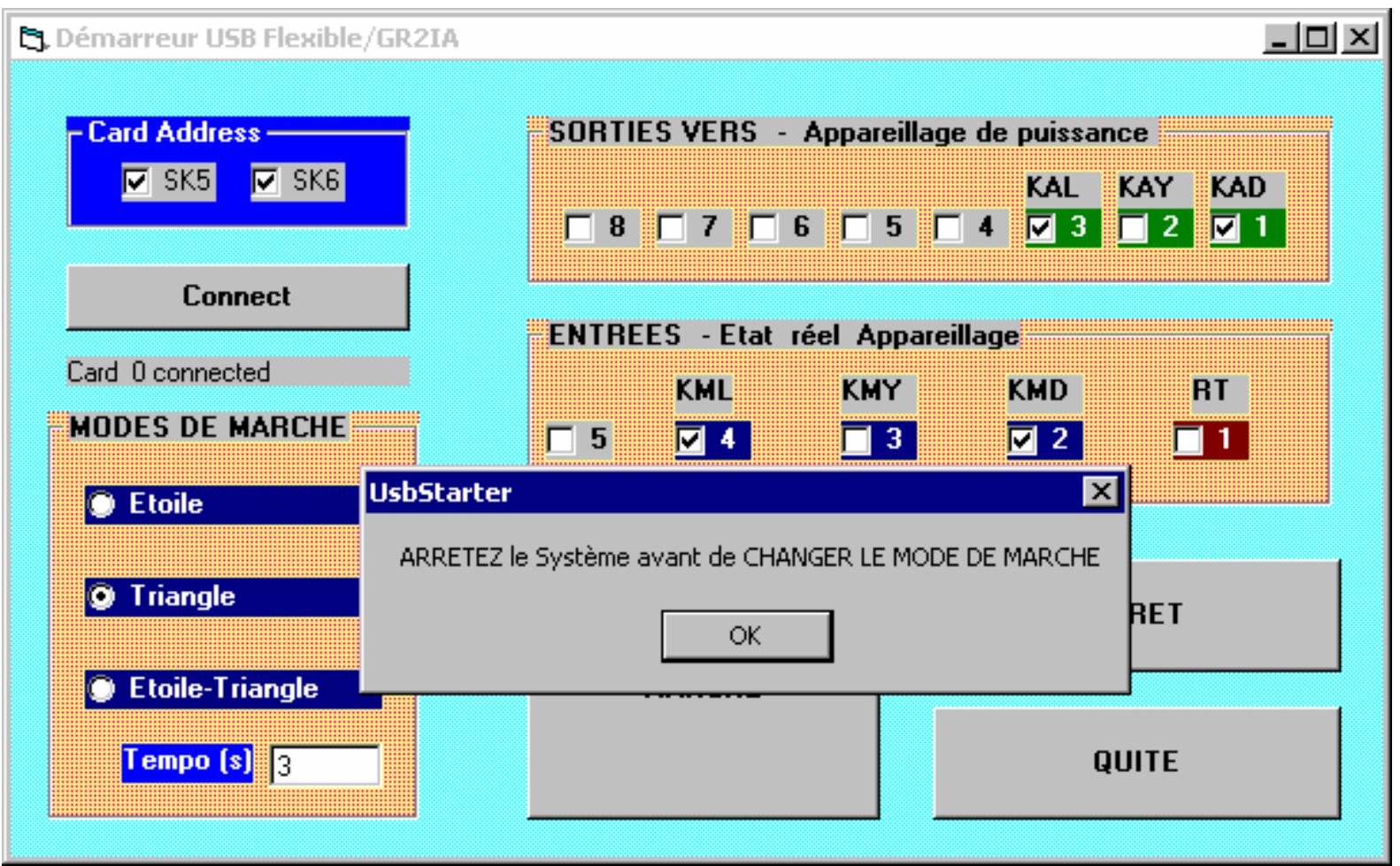

Figure 9 Changement de mode de démarrage sécurisé 


\section{Biographie des auteurs :}

1) J. МВIHI a obtenu le Ph.D. en génie électrique et informatique à l'École Polytechnique de Montréal (Québec, Canada) en 1999. Il est actuellement enseignant-chercheur, responsable du Groupe de Recherche en Informatique Industrielle et Automation (GR2IA), et chef du département d'Industries Textiles et de l'Habillement à l'ENSET de Douala. Il est membre du comité scientifique de l'unité de formation doctorale en Physique et Sciences de l'Ingénieur de l'Université de Douala, et membre du Comité Consultatif des Institutions Universitaires du Cameroun. Il est auteur de l'ouvrage Informatique et Automation, publié aux éditions Publibook de Paris (2006). Ses recherches actuelles portent sur les nouveaux systèmes d'instrumentation virtuelle et d'automation locale ou à distance.

2) $\boldsymbol{E}$. $\boldsymbol{A M I E} \boldsymbol{E B} \boldsymbol{A} \boldsymbol{N D} \boldsymbol{A}$ est titulaire du Diplôme de Professeur d'Enseignement Technique (DIPET) de $2^{\mathrm{e}}$ grade en électrotechnique, obtenu à l'ENSET de Douala au Cameroun. Dans le cadre de son mémoire de fin d'étude pour l'obtention de son DIPET 2, il a participé en 2006 au sein du GR2IA aux travaux d'étude, de montage et de mise en service de la partie interface USB présenté dans cet article.

3) A. DONWOUNG KANA est titulaire du Diplôme de Professeur d'Enseignement Technique (DIPET) de $2^{\mathrm{e}}$ grade en électrotechnique, obtenu à l'ENSET de Douala au Cameroun. Dans le cadre de son mémoire de fin d'étude pour l'obtention de son DIPET 2, il a participé en 2006 au sein du GR2IA aux travaux d'étude, de montage et de mise en service de la partie interface USB présenté dans cet article. 